\title{
Rapid Determination of Methicillin Resistance among Staphylococcus aureus Clinical Isolates by Colorimetric Methods
}

\section{Ahmet Yilmaz Coban}

Ondokuz Mayis University, Medical School, Department of Medical Microbiology, Samsun, Turkey

Volume 50, no. 7, p. 2191-2193, 2012. Page 2191, column 1, lines 12-14: "Furthermore, the mortality rate is higher in methicillinsusceptible S. aureus (MSSA) septicemia than MRSA septicemia" should read "Furthermore, the mortality rate is higher in MRSA septicemia than methicillin-susceptible S. aureus (MSSA) septicemia." 